\title{
LA FUNCIÓN DE LA INVESTIGACIÓN EN EL PROCESOACADÉMICO DE LA ESCUELA DE TRABAJO SOCIAL
}

Ivette Campos Moreira'

\section{Introducción}

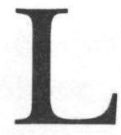

a actualización permanente del conocimiento es la base para el desarrollo y consolidación de una disciplina. En este sentido, la investigación se constituye en el medio que permite la renovación permanente del conocimiento, la actualización y la legitimidad de la disciplina. La esencia del Trabajo Social contempla la condición descrita como inherente a su naturaleza. De ahí, que es de gran valor la reflexión de la función que se le confiere a la investigación en la disciplina del Trabajo Social. Este artículo presenta mis reflexiones en torno a la función de la investigación en la Escuela de Trabajo Social y a los espacios sociales que favorecen el desarrollo en la producción del conocimiento en esta Unidad Académica.

\section{La función de la investigación}

La investigación es concebida como uno de los pilares que permiten la permanente actualización del conocimiento y también la generación de éste. El desarrollo de la investigación es de vital importancia en las unidades formadoras que tienen como premisa \footnotetext{
Rica.

${ }^{1}$ Profesora Asociada de la Escuela de Trabajo Social, Universidad de Costa
} 
fundamental el conocimiento para la intervención, que es la situación particular de la disciplina de Trabajo Social.

La investigación en nuestra unidad académica ha constituido a lo largo de su desarrollo histórico, uno de los pilares fundamentales, junto con la docencia y con la acción social. Al respecto, la investigación cumple funciones sustantivas en el quehacer académico como fuente de conocimiento, como apoyo a la docencia y a la acción social. El compromiso con el estudio de problemas sociales; el análisis y el planteamiento de políticas sociales que mejoren la calidad de vida de la sociedad costarricense en el marco de los derechos humanos, se constituyen en el objeto de estudio e intervención de la academia.

La función de la investigación es planteada entonces, como aquella actividad vital que contribuye al análisis, a la interpretación y a la transformación de los problemas sociales que constituyen la demanda social que justifica la definición, la reorientación y el fortalecimiento de políticas sociales.

La naturaleza del Trabajo Social como profesión, se caracteriza por su orientación a la práctica, hacia la intervención en situaciones sociales concretas. Esto, define el rol o papel profesional de quiénes practican el Trabajo Social, deben ocuparse de la solución de problemas sociales, interesándose por el conocimiento que puede ser transformado en acciones intencionadas que propicien el cambio social. En este sentido, el Trabajo Social puede ser conceptualizado como la disciplina donde la investigación se constituye en el fundamento de intervenciones sociales y toma de decisiones. La función esencial de la investigación descansa en la solución de problemas, el esclarecimiento y orientación hacia cambios necesarios. Por ello, debe desembocar en la producción de conocimientos, el manejo riguroso y sistemático de la información que sea útil para el cambio social. En este sentido la conceptualización de la investigación (Guzmán, 1997) permite caracterizarla como la pesquisa sistemática que utiliza una metodología rigurosa y flexible para la resolución de problemas y crear un conocimiento que generalmente se aplica a situaciones concretas. Este nuevo acervo de conocimientos debe enriquecer la base de conocimiento disciplinario y potenciar el desarrollo disciplinario.

Existen varios roles en el quehacer investigativo:

- Uno de ellos es el de consumidor de investigaciones. Este se fundamenta en el conocimiento existente como plataforma 
para la intervención profesional. Este rol obliga a un aprendizaje en la búsqueda de identificación de estudios y publicaciones apropiados para determinar la validez y confiabilidad del conocimiento necesario que se traduzca en técnicas de intervención para la práctica académica.

- Otro rol es el de diseminador de los resultados de la información a la comunidad de estudiantes, profesores, sujetos participantes, profesionales, de manera que este acervo de investigación esté disponible a la comunidad de Trabajadores Sociales.

- Otro rol importante es el de colaborador o asociado a otros esfuerzos investigativos cuyos problemas de investigación son similares. En este caso, el impacto de los resultados es mayor. En este sentido, profesores y estudiantes establecen relaciones de cooperación por medio de proyectos de investigación; propicia la discusión de ideas e inquietudes sobre los problemas de estudio, las experiencias, logros y frustraciones durante los procesos de investigación; el desarrollo de destrezas que le permitan criticar efectiva y constructivamente el trabajo propio y el de otros y el logro de una visión más comprensiva.

- Y por último, el rol de producción o creación de conocimiento nuevo. La naturaleza de la disciplina y la profesión le otorga a la investigación un papel vital en la generación de conocimiento nuevo. El proceso que se sigue en la formación académica perfila dos vertientes: Una ligada a la comprensión del contexto como estructura emergente de situaciones sociales pertinentes al trabajo social y el otro ligado a la comprensión, al análisis y a las transformaciones. Este rol sintetiza la esencia de la investigación en el sentido, de condensar los roles anteriores y a la vez trascenderlos. Sin embargo, esta condición no excluye que existan experiencias de investigación que privilegien uno o dos roles señalados en el sentido de que se constituyan en parte de procesos académicos más amplios donde la conjugación de los múltiples esfuerzos contribuyen a la producción del conocimiento. 


\section{Espacios sociales para el desarrollo de la producción de conocimiento}

La legitimidad de una disciplina está fundamentada en la necesidad de satisfacer las demandas sociales. Ante esto, los espacios que emergen como prioritarios y puntos de partida para realizar investigaciones son los que aportan información sobre las necesidades sociales de los distintos grupos de población. Es importante la distinción por grupos etáreos, por género, por condición económi$\mathrm{ca}$, por la capacidad que demuestren para dar respuesta a sus necesidades. A modo de ilustración, la Escuela de Trabajo Social cuenta con una forma muy particular de captación de las necesidades sociales. Esta se caracteriza por la explicitación de "áreas críticas" que se conciben como rutas de conocimiento y de intervención que se constituyen en puntos de partida para el quehacer investigativo de la Unidad Académica.

A partir del quinquenio 1995-2000, se plantean seis áreas denominadas:

- "De la pobreza a la calidad de vida en igualdad de oportunidades"

- "Del deterioro de la salud a la salud integral"

- "Del deterioro del ambiente hacia el desarrollo sostenible"

- "De la crisis del Estado de Bienestar hacia la reforma del Estado"

- "De la violencia social a la democratización de la vida cotidiana y la convivencia social"

- "Del autoritarismo y la exclusión a la participación social y la ciudadanía plena" (Sección de Investigación, Escuela de Trabajo Social, 1995).

El planteamiento de cada área crítica, identifica la problemática social donde se focaliza la acción investigativa y la intervención en cada una de ellas se precisan los caminos que iluminan sobre la respuesta ante el problema social y la aspiración en la atención a éste. Por ello se da prioridad a la participación ciudadana y estatal en cada una de áreas descritas. Esta participación incluye la distinción por grupo etáreo, por género, por condición económica. 
En este sentido, una disciplina que no despliegue acciones investigativas en relación con el conocimiento, la reflexión, la intervención en el entorno en que se halla inserta tiene grandes posibilidades de estancamiento y a largo plazo a su extinción. Este autismo ante las necesidades sociales del país podría tomar el rumbo descrito. Por ello la investigación debe concebirse como un proceso en constante lucha con los formalismos que la hagan obsoleta; que no la condenen al consumismo de investigaciones preexistentes y que sus aspiraciones trasciendan a objetivos que conduzcan a la búsqueda de la verdad, la justicia, los derechos humanos.

\section{La producción de conocimiento en la Escuela de Trabajo Social}

Con el propósito de realizar una disquisición en torno a los estudios que se producen en el proceso académico de profesores y estudiantes se encuentran dos vertientes predominantes: una ligada a los estudios que emergen de los cursos que tienen como actividad de aprendizaje esencial la realización de investigaciones. Se persigue en este campo formar practicantes investigadores, a partir de prácticas guiadas de investigaciones cualitativas, cuantitativas que puedan dar explicaciones, interpretaciones epistemológicas a las intervenciones que demanda la sociedad actual. Destacan los estudios que se realizan en los cursos denominados Talleres, Seminarios y los Trabajos Finales de Graduación. ${ }^{2}$

La investigación en el pensum actual, subraya el estudio de las problemáticas sociales y sus manifestaciones en grupos etáreos de mayor riesgo. Como complemento, a esta problemática, se han producido estudios en la dimensión preventiva, en el sentido de plantear investigaciones y acciones de intervención en relación con alternativas de protección a niñas/os y adolescentes en riesgo social. Estas posibilidades de acción, se han focalizado en los sistemas locales y Juntas de Protección a la Infancia. Otro conjunto de investigaciones de relevancia, privilegia, la gestión local del desarrollo y del riesgo. En éstas interesa profundizar la función social de las municipalidades y en éstas el papel de las Oficinas de la Mujer. La participación

\footnotetext{
${ }^{2}$ Estas investigaciones se originan en dos cursos denominados Diseño de Trabajo Final de Graduación. En este sentido se toman éstos ya que constituyen su culminación.
} 
social y la organización institucional en torno a la construcción de servicios de salud, de empleo, de administración de la justicia, es otro conglomerado de investigaciones que aportan conocimientos a estudiantes y profesores.

La otra vertiente provee actividades de aprendizaje que privilegian parte del proceso de investigación; no es su objetivo el seguimiento formalizado de un proceso completo. Destacan cursos que proveen información, resúmenes, análisis de otros autores en torno a aspectos relevantes de la disciplina. Destacan en este conjunto los cursos Trabajo Social I y II; Epistemología; Ideología, Etica y Derechos Humanos, Organizaciones y Movimientos Sociales, Trabajo Social y Familia; Sistemas de Atención Social; Teoría del Estado y Política Social, Planificación y Programación Social. Sin embargo, en estos cursos no se exime la incorporación de actividades de aprendizaje que posibiliten el contacto con las realidades particulares del objeto de estudio de los cursos, sólo que éstas cumplen propósitos demostrativos e ilustrativos. El cuadro $\mathrm{N}^{\circ} 1$, sintetiza lo señalado.

Otra forma de visibilizar los estudios que se producen en nuestra Unidad Académica, se presenta en el Cuadro № 2, donde se destacan la distribución de los estudios, según las áreas críticas. Ello nos ofrece desde otra óptica el contenido del conocimiento que se privilegia en la elaboración de estudios. 
CUADRO $\mathrm{N}^{\circ} 1$

Tipo de conocimiento que se produce en la Escuela de Trabajo Social AÑO 2000

\begin{tabular}{|c|c|c|}
\hline CURSO & TIPO DE CONOCIMIENTO & $\begin{array}{c}\text { ROLES } \\
\text { PREDOMINANTES }\end{array}$ \\
\hline $\begin{array}{l}\text { Taller I Problemas } \\
\text { Macro-Microsociales }\end{array}$ & $\begin{array}{l}\text { Sobre la base de fuentes bibliográficas } \\
\text { existentes se conoce sobre violencia doméstica, } \\
\text { desempleo, pobreza, la atención profesional. }\end{array}$ & $\begin{array}{l}\text { Consumidores de } \\
\text { investigaciones }\end{array}$ \\
\hline $\begin{array}{l}\text { Taller II Métodos para la } \\
\text { Investigación Diagnóstica } \\
\text { en Organizaciones y } \\
\text { Grupos } \\
\text { Taller III Intervención con } \\
\text { Grupos, Organizaciones y } \\
\text { Comunidades }\end{array}$ & $\begin{array}{l}\text {-Se hace diagnósticos en comunidades: sobre } \\
\text { sus principales necesidades y las posibilidades } \\
\text { de participación de grupos. Comités Tutelares } \\
\text { de Menores, Municipalidades y Comités de Salud } \\
\text { (Curridabat, Desamparados, San Carlos) } \\
\text {-Vivencia y aplicación de estrategias de } \\
\text { organización y capacitación con los grupos }\end{array}$ & $\begin{array}{l}\text { Creadora y } \\
\text { diseminadora de } \\
\text { conocimiento } \\
\text { Asociado } 0 \\
\text { colaborador }\end{array}$ \\
\hline $\begin{array}{l}\text { Taller IV y V Trabajo Social } \\
\text { Institucional }\end{array}$ & $\begin{array}{l}\text {-Conocimiento a partir de la inmersión en el trabajo } \\
\text { cotidiano de los sistemas locales de protección } \\
\text {-Trabajo Social en el PANI, en la CCSS, Ministerio } \\
\text { de Justicia, Programa de Atención Integral de Salud; } \\
\text { Montes de Oca - Curridabat, Municipalidades, } \\
\text { Comisión Nacional de Emergencia, Ministerio de } \\
\text { Educación Pública }\end{array}$ & $\begin{array}{l}\text { Creadoras y } \\
\text { diseminadoras de } \\
\text { conocimiento } \\
\text { Asociado }\end{array}$ \\
\hline $\begin{array}{l}\text { Taller VI y VII Intervención } \\
\text { en Programas de } \\
\text { Trabajo Social }\end{array}$ & $\begin{array}{l}\text { Estudio de políticas en torno a: } \\
\text {-Políticas públicas y fortalecimiento en núcleos } \\
\text { familiares } \\
\text {-Programas de atención de niños en riesgo social en } \\
\text { San Carlos } \\
\text {-Formas organizativas alternas para el caso de } \\
\text { organizaciones no gubernamentales vinculadas al } \\
\text { empleo } \\
\text {-Municipalidad/Gestión local del desarrollo } \\
\text {-Programa de Atención Integral de salud } \\
\text { (Montes de Oca y Curridabat) }\end{array}$ & $\begin{array}{l}\text { Creadoras y } \\
\text { diseminadoras de } \\
\text { conocimiento } \\
\text { Asociada }\end{array}$ \\
\hline Trabajo Social I & $\begin{array}{l}\text {-Origen y desarrollo histórico del Trabajo Social y } \\
\text { sus particularidades en Europa, América Latina y } \\
\text { Costa Rica } \\
\text {-Objeto, objetivos/campos de acción del Trabajo } \\
\text { Social en Costa Rica }\end{array}$ & $\begin{array}{l}\text { Consumidor de } \\
\text { investigación y del } \\
\text { relato y de la } \\
\text { experiencia de los } \\
\text { trabajadores sociales } \\
\text { en los distintos } \\
\text { campos del } \\
\text { Trabajo Social }\end{array}$ \\
\hline Trabajo Social y Población & $\begin{array}{l}\text { Principales indicadores demográficos y } \\
\text { socioeconómicos como herramientas para el Trabajo } \\
\text { Social en general y su expresión en pobreza, salud }\end{array}$ & $\begin{array}{l}\text { Consumidores de } \\
\text { investigaciones }\end{array}$ \\
\hline
\end{tabular}




\begin{tabular}{|c|c|c|}
\hline CURSO & TIPO DE CONOCIMIENTO & $\begin{array}{c}\text { ROLES } \\
\text { PREDOMINANTES }\end{array}$ \\
\hline $\begin{array}{l}\text { Espistemología del Trabajo } \\
\text { Social I, II y III }\end{array}$ & $\begin{array}{l}\text { Caracterización de las corrientes epistemológicas de } \\
\text { mayor influencia en el desarrollo del Trabajo Social }\end{array}$ & $\begin{array}{l}\text { Consumidores del } \\
\text { conocimiento } \\
\text { acumulado }\end{array}$ \\
\hline $\begin{array}{l}\text { Ideología, Ética y Derechos } \\
\text { Humanos }\end{array}$ & $\begin{array}{l}\text {-Sobre las relaciones entre los principios de la ética, } \\
\text { ideología y derechos humanos y Trabajo Social } \\
\text {-El fenómeno de la discriminación en sus distintas } \\
\text { manifestaciones en las relaciones humanas y en la } \\
\text { teoría y práctica del Trabajo Social }\end{array}$ & $\begin{array}{l}\text { Consumidores de } \\
\text { Conocimiento }\end{array}$ \\
\hline $\begin{array}{l}\text { Teoría y Metodología para } \\
\text { el Trabajo en Comunidad }\end{array}$ & $\begin{array}{l}\text { Diversos conceptos, enfoques y métodos del Trabajo } \\
\text { Social en comunidad }\end{array}$ & $\begin{array}{l}\text { Consumidores y la } \\
\text { aplicación de este } \\
\text { conocimiento }\end{array}$ \\
\hline Teoría y Método de Grupo & $\begin{array}{l}\text {-Sobre el potencial del grupo como medio para el } \\
\text { crecimiento y modificación de conductas de } \\
\text { personas } \\
\text { existente } \\
\text {-Y como medio para el logro de tareas } \\
\text {-Diferentes enfoques metodológicos para la } \\
\text { intervención de grupos }\end{array}$ & $\begin{array}{l}\text { Consumidores del } \\
\text { Conocimiento } \\
\text { Aplicación de } \\
\text { técnicas para el } \\
\text { trabajo con grupos }\end{array}$ \\
\hline $\begin{array}{l}\text { Promoción Humana y } \\
\text { Animación Sociocultural }\end{array}$ & $\begin{array}{l}\text { Enfoques epistemológicos y metodológicos en los } \\
\text { procesos de promoción humana, la educación } \\
\text { popular y animación sociocultural }\end{array}$ & $\begin{array}{l}\text { Consumidores y } \\
\text { aplicación de } \\
\text { técnicas } \\
\text { en este campo }\end{array}$ \\
\hline $\begin{array}{l}\text { Organizaciones y } \\
\text { Movimientos Sociales } \\
\text { de Costa Rica }\end{array}$ & $\begin{array}{l}\text { Dinamismo social, político e ideología sobre el origen } \\
\text { y desarrollo del movimiento popular costarricense }\end{array}$ & $\begin{array}{l}\text { Consumidores de } \\
\text { conocimiento }\end{array}$ \\
\hline Trabajo Social y Familia & $\begin{array}{l}\text { Tres modelos de terapia familiar sistémica como } \\
\text { base para la intervención terapéutica }\end{array}$ & Consumidores \\
\hline $\begin{array}{l}\text { Sistemas de Atención } \\
\text { Social Institucional }\end{array}$ & $\begin{array}{l}\text { Sobre los sistemas de atención social institucional } \\
\text { de acuerdo con problemáticas y áreas de } \\
\text { participación real y posible del Trabajo Social }\end{array}$ & $\begin{array}{l}\text { Consumidores de } \\
\text { conocimiento }\end{array}$ \\
\hline $\begin{array}{l}\text { Teoría del Estado y } \\
\text { Política Social I y II }\end{array}$ & $\begin{array}{l}\text { Enfoques teóricos del Estado y la política, sobre las } \\
\text { reformas y experiencias en América Latina y } \\
\text { Costa Rica }\end{array}$ & Consumidores \\
\hline $\begin{array}{l}\text { Planificación y } \\
\text { Programación Social I y II }\end{array}$ & $\begin{array}{l}\text { Premisas teóricas, epistemológicas de los procesos } \\
\text { participativos de planificación y gestión local desde } \\
\text { el Trabajo Social }\end{array}$ & Consumidores \\
\hline
\end{tabular}


(continuación Cuadro No1)

\begin{tabular}{|c|c|c|}
\hline CURSO & TIPO DE CONOCIMIENTO & $\begin{array}{c}\text { ROLES } \\
\text { PREDOMINANTES } \\
\end{array}$ \\
\hline $\begin{array}{l}\text { Supervisión en Trabajo } \\
\text { Social }\end{array}$ & $\begin{array}{l}\text { Fundamentos teóricos, epistemológicos de la } \\
\text { supervisión como proceso que coadyuva a la calidad } \\
\text { de servicios }\end{array}$ & Consumidores \\
\hline $\begin{array}{l}\text { Sistematización en Trabajo } \\
\text { Social }\end{array}$ & $\begin{array}{l}\text { Aspectos teóricos, epistemológicos y puesta en } \\
\text { práctica de la sistematización del Trabajo Social } \\
\text { en Costa Rica. Sistematización del aspecto de la } \\
\text { práctica }\end{array}$ & $\begin{array}{l}\text { Consumidores de } \\
\text { investigación } \\
\text { Aplicación }\end{array}$ \\
\hline $\begin{array}{l}\text { Métodos de Intervención } \\
\text { en Microsocial I y II }\end{array}$ & $\begin{array}{l}\text { El sustento teórico, metodológico del Método } \\
\text { de Caso }\end{array}$ & $\begin{array}{l}\text { Consumidores } \\
\text { Aplicación }\end{array}$ \\
\hline Seminario I y II & $\begin{array}{l}\text {-Definición de temas y problemas relevantes } \\
\text { para el Trabajo Social } \\
\text {-Discapacidad, violencia doméstica, menores } \\
\text { de edad, adolescentes en situaciones difíciles, } \\
\text { población con SIDA, adultos }\end{array}$ & $\begin{array}{l}\text { Consumidores } \\
\text { Aplicación }\end{array}$ \\
\hline $\begin{array}{l}\text { Investigación Evaluativa } \\
\text { | y || }\end{array}$ & $\begin{array}{l}\text {-La evaluación en el contexto económico, social, } \\
\text { político } \\
\text {-Prediseño de evaluación para el servicio en } \\
\text { que se inscribe la práctica }\end{array}$ & $\begin{array}{l}\text { Consumidores } \\
\text { Aplicación }\end{array}$ \\
\hline $\begin{array}{l}\text { Diseño de Trabajo Social } \\
\text { I y || }\end{array}$ & $\begin{array}{l}\text { Elaboración del proceso de investigación de } \\
\text { problemas sociales e institucionales }\end{array}$ & $\begin{array}{l}\text { Creación y } \\
\text { diseminación } \\
\text { de información }\end{array}$ \\
\hline $\begin{array}{l}\text { Administración de } \\
\text { Programas }\end{array}$ & $\begin{array}{l}\text {-Módulos de gestión en los servicios } \\
\text { público/privados } \\
\text {-Elaboración de propuestas metodológicas } \\
\text { para la conducción de los servicios }\end{array}$ & $\begin{array}{l}\text { Consumidores } \\
\text { Aplicación }\end{array}$ \\
\hline
\end{tabular}

Fuente: Programas I y II ciclo 2000. Secretaría de Docencia. Escuela de Trabajo Social, Sección de Docencia. Universidad de Costa Rica, Sede Rodrigo Facio. 2000. 


\begin{tabular}{|c|c|}
\hline \multicolumn{2}{|c|}{$\begin{array}{c}\text { CUADRO } \mathrm{N}^{\circ} 2 \\
\text { Principales investigaciones realizadas por estudiantes } \\
\text { según Áreas Críticas (1999-2001) }\end{array}$} \\
\hline $\begin{array}{l}\text { - "De la pobreza a la calidad de vida } \\
\text { en igualdad de oportunidades" }\end{array}$ & $\begin{array}{l}\text { Estudios en comunidades urbano rurales de } \\
\text { atención prioritaria en la educación pública }\end{array}$ \\
\hline $\begin{array}{l}\text { - "Del deterioro de la salud a la salud } \\
\text { integral" }\end{array}$ & $\begin{array}{l}\text { - Participación social en Salud. EBAIS y } \\
\text { Comités de Salud. } \\
\text { - Atención en Salud, Clínicas, Hospitales. } \\
\text { - Programa de Atención Integral en Salud. } \\
\text { Cantones Montes de Oca y Curridabat. }\end{array}$ \\
\hline $\begin{array}{l}\text { - Del deterioro del ambiente hacia el } \\
\text { desarrollo sostenible" }\end{array}$ & No hay \\
\hline $\begin{array}{l}\text { - "De la crisis del Estado de Bienestar hacia } \\
\text { la reforma del Estado" }\end{array}$ & $\begin{array}{l}\text { Formas organizativas alternativas para la } \\
\text { construcción y producción de servicios } \\
\text { sociales y productivos. El caso No } \\
\text { Gubernamen tales a la producción de } \\
\text { empleo de las organizaciones. }\end{array}$ \\
\hline $\begin{array}{l}\text { - "De la violencia social a la } \\
\text { democratización de la vida cotidiana y la } \\
\text { convivencia social" }\end{array}$ & $\begin{array}{l}\text { Estudios sobre la niñez: Protección de } \\
\text { derechos y los Comités Tutelares de Menores. } \\
\text { Niñez y Adolescencia. Protección de Derechos. } \\
\text { Sistemas Sociales de protección a la Niñez. } \\
\text { Juntas Cantonales de Protección. Las } \\
\text { políticas públicas y fortalecimiento de núcleos } \\
\text { familiares democráticos. Atención de niños } \\
\text { en riesgo social. }\end{array}$ \\
\hline $\begin{array}{l}\text { - "Del autoritarismo y la exclusión a la } \\
\text { participación social y la ciudadanía } \\
\text { plena". }\end{array}$ & $\begin{array}{l}\text { Municipalidades: Gestión local del desempleo. } \\
\text { Gestión local del riesgo. } \\
\text { Oficinas de la Mujer. } \\
\text { Comisión Nacional de Emergencia. } \\
\text { Comisión Nacional de Salud. }\end{array}$ \\
\hline
\end{tabular}

Fuente: Elaboración propia a partir de los documentos de las Áreas Críticas, Correspondencia a profesores sobre Centros de Práctica. 2001. Plan de Estudios. 


\section{La investigación desde los trabajos finales de graduación (T.F.G.)}

El conocimiento que se ha generado desde esta vertiente, ha aportado una diversidad de conocimiento en variados espacios de interés para la disciplina y la profesión del Trabajo Social. Un estudio que se registra en 1997 plantea como temáticas relevantes las que se refieren al estudio de las condiciones de vida. Otro conjunto de trabajos, ligados a salud, y a problemáticas sociales, se constituyen en el conocimiento que permite estimar la prevalencia de los tópicos estudiados en un período de 45 años.

Ahora bien, con una menor frecuencia se destacan otros temas, como el conocimiento del papel de los grupos sociales sujetos de la intervención del Trabajo Social. Otros ligados a los procesos sociales relevantes para la profesión como pueden ser la participación social, el desarrollo social. En menor medida, sobresalen ligados al ejercicio profesional, y a los recursos humanos.

El cuadro $\mathrm{N}^{\circ} 3$, que a continuación se expone presenta la frecuencia de los temas que se investigaron en un período de 45 años, desde 1952 hasta el año 1991.

\section{CUADRO $\mathrm{N}^{\circ} 3$ \\ TFG Según descriptores temáticos 1952-1957}

\begin{tabular}{|l|l|}
\hline Descriptores temáticos & No $^{\circ}$ desis \\
\hline Salud & 64 \\
Condición de Vida & 80 \\
Grupos Sociales & 46 \\
Procesos sociales & 35 \\
Problemáticas Sociales & 63 \\
Recursos Humanos & 17 \\
Ejercicio Profesional & 21 \\
Varios Temas & 18 \\
\hline Total & $\mathbf{3 4 4}$ \\
\hline
\end{tabular}

Fuente: Anexo №3. Áreas Críticas. Sección de Investigación. 1997. Archivos Sección de Investigación. 1995-2000. Escuela de Trabajo Social. 2001 
Tal codificación ofrece información sobre las prioridades que emergen de la práctica investigativa en los TFG. La necesidad de realizar estudios contextuales, las condiciones de vida ligadas a la pobreza, marginalidad, al empleo, subempleo y a las problemáticas sociales, marcan la tendencia que caracterizó el ejercicio de la profesión en ese período. Sin embargo, es importante subrayar, en este conjunto de investigaciones, la función de éstas en el proceso formativo y en la necesidad social que atiende. Al respecto, las investigaciones realizadas podrían perderse en una suma de datos de diversa naturaleza y especificidad. El agravante de esto es su relativa incapacidad para tomar decisiones con respecto al aporte de estos estudios al conocimiento para la definición de áreas geográficas de impacto, instituciones y programas sociales.

La posibilidad de "potencializar el conocimiento producido en forma atomizada, generó la necesidad de plantear "síntesis del conocimiento", denominadas "Areas Críticas" para el despliegue de acciones de investigación de docencia y de acción social. Esta se formalizó en 1997. Estas áreas criticas proveen un valioso punto de arranque.

\section{Las Áreas Críticas como punto de partida para la identifica- ción de temas de los Trabajos Finales de Graduación}

La experiencia investigativa de los TFG, su origen y desarrollo identificado en un lapso de 1952-1997, marcó un avance en el conocimiento de problemáticas específicas, de naturaleza variada y que se constituía en bagaje de conocimiento desarticulado, lo que impide conocer el impacto social de las investigaciones en tanto los cambios que podían generar en el comportamiento de los grupos o en las políticas sociales. Sin embargo, su variedad conlleva intrínseca su vulnerabilidad, en el sentido de constituir un conglomerado de temas sin conexión, donde priva como único criterio el interés del sustentante, único criterio para el estudio de un tema. La atomización de estudios no permite captar el avance en el conocimiento de la realidad como ya se ha dicho.

La yuxtaposición de la realización de Trabajos Finales de Graduación en relación con las áreas críticas permiten visualizar una tendencia a tratar temáticas dirigidas a la calidad de vida de los grupos etáreos, de las familias, de las madres, de las adolescentes, se constituyen en aspectos relevantes considerados por los estudian- 
tes para el enriquecimiento de la formación académica. Si se compara con los datos que se presentan en el período 1952-97, hay coincidencia en el interés por el estudio de las condiciones de vida de los distintos grupos "sujetos de estudio" de la formación académica. Se deriva una intencionalidad manifiesta por realizar estudios que aporten a los aspectos positivos que despliegan los grupos, las personas para el mejoramiento de la calidad de vida. En este sentido, sobresalen temas ligados a la Resiliencia (Badilla y Sancho, 1996); a los factores protectores y la calidad de vida de adultos mayores (Barrantes, Marín y Murillo, 1998); adolescentes (Carballo et al., 1998) la recreación en los jóvenes (Moreno, 2000); la aplicación de modelos ligados al manejo de la inteligencia emocional (Ramírez, Montenegro y Ramírez, 2001). 


\section{CUADRO $\mathrm{N}^{\circ} 4$ \\ Clasificación de Trabajos Finales de Graduación \\ 1998-2000}

De la calidad de vida en igualdad de oportunidades

Del deterioro de la salud

a la salud integral.

Del deterioro del ambiente al desarrollo sostenible.
- Factores protectores

- Calidad de vida y de los adultos

- La jubilación en la empresa privada DEMASA

- La deserción escolar y la relación de los niños trabajadores.

- Cotidianidad de las familias en las fincas bananeras.
- Vínculos afectivos entre madres e hijos. Albergue El Buen Pastor.

- Menor trabajador adolescente en Liberia.

- Derechos sociales de la población migrante.

- La recreación de la adolescente en Liberia.

- Adolescentes en prostitución.

- Unidos construyendo una ilusión.

- La coordinación interinstitucional como respuesta a la población indigente.
- Escuelas de Salud

- Modelos de Gestión y atención en la Asociación Guanacasteca de Desarrollo Forestal.

- Formas organizativas alternativas para el caso de las organizaciones no gubernamentales vinculadas al empleo.

- Municipalidades. Gestión local del desarrollo.

- Programa de Atención Integral de Salud de Montes de Oca y Curridabat. 


\section{Lecciones Aprendidas}

En síntesis, la investigación es parte integral del conjunto articulado de cursos que conforman el plan de estudios. Esta característica se evidencia en la inclusión de la investigación como uno de los hilos conductores del curriculum.

Los cursos, tanto los "teóricos" como los "prácticos" 3 contemplan en su contenido distintas formas de investigación. Desde la revisión de textos y comprensión de conceptos, hasta estudios donde el método científico es parte integral de la intervención. El resultado de estos estudios se cristaliza como conocimientos que se acercan a la realidad social, al análisis de las políticas y servicios sociales y sobre todo al examen crítico del quehacer profesional. La investigación no sólo debe ser un método para realizar estudios científicos propiamente dichos, sino que debe ser una forma de pensar la realidad, en forma organizada sistemática y crítica. La culminación o propósito intrínseco de lo anterior se debe traducir en la transformación propositiva del quehacer profesional, de su impacto al entorno y de las posiciones epistemológicas teóricas que deben y pueden sustentar el devenir de la profesión y la disciplina.

\section{Principales aspectos críticos}

La práctica investigativa tiene su expresión concreta en el plan de estudios, en los talleres, en los cursos instrumentales, los Seminarios se constituyen en los núcleos "potenciales" para la producción de conocimiento nuevo. En este sentido, estudiantes, profesores, profesionales, personas. Incursionan en la aventura de conocer, de explicitar procesos que vivencian los grupos, las familias, los profesionales para plantear soluciones, descubrir, entender y explicar las situaciones que afectan e inciden en la calidad de vida de las personas. Sin embargo, pese a un conjunto importante de estudios en la generación de cambios en beneficio de las poblaciones afectadas.

El compromiso que se explicita en la misión y visión (Escuela de Trabajo Social, 2001) debe ser un Norte; sin embargo, esto no es

${ }^{3}$ Estos términos son acuñados por la comunidad estudiantil y por el profesorado, para referirse a los cursos que procuran objetivos referidos a los niveles de información y a la comprensión en el primer caso. En el segundo, referidos a los que implican una intervención del estudiante en la atención de problemas sociales. 
visible en los resultados de la investigación ya que hay una tendencia a realizar estudios cuyo conocimiento se queda en el estudiante o en las bibliotecas. En el caso de estudios que posibiliten un cambio en los actores estudiantiles, comunales, profesionales, no hay manera de constatar éstos ya que no hay una evaluación permanente de ello.

- Pese a los estudios de problemáticas en torno a la niñez y la incidencia de éstos sigue aumentando.

- Se requiere de un esfuerzo articulado; y lo más importante es la participación de los sujetos con posturas críticas y con ello la transformación de las situaciones sociales. De esta manera la investigación no sólo cumple una función reproductora de un conocimiento que no genera cambios, donde la transformación, como objetivo social sería sólo parte del discurso.

- Es necesario volver a otras aristas no sólo estudiar los problemas, sino las potencialidades, los recursos personales que tienen las personas. De allí que es fundamental volver la mirada a estudios sobre la solidaridad, las potencialidades de los sujetos, el ejercicio de los derechos humanos por los distintos grupos.

- Las investigaciones no deben concentrarse en espacios concretos en el área metropolitana; deben incluir otras áreas rurales. La atomización y descontextualización que caracteriza a los estudios conlleva a una sumatoria de conocimientos. La capitalización de éstos en nuestra unidad académica para la toma de decisiones, es muy limitada.

- Por ello es importante crear políticas de "articular esfuerzos para potencializar el conocimiento que se produce. Si bien es cierto hay refuerzos en este sentido en el 2001 es necesario crear actividades de evaluación para recuperar información en torno al impacto que están produciendo las investigaciones que se producen en la Escuela de Trabajo Social (Casas, 2001). 


\section{Referencias bibliográficas}

Badilla, H. y Sancho, A. (1998). Resiliencia y Trabajo Social. Tesis no publicada, Escuela de Trabajo Social, Universidad de Costa Rica.

Barrantes, A.; Marín, M. y Murillo, Flor María (1998). Factores sociales protectores de los adultos(as) y su relación con la calidad de vida. Tesis no publicada, Escuela de Trabajo Social, Universidad de Costa Rica.

Carballo, Ana I.; Hernández, G.; Elizondo, J.; Rodríguez, G. y Serrano, X. (1998). El proyecto de vida desde la perspectiva de los jóvenes. Tesis no publicada, Escuela de Trabajo Social, Universidad de Costa Rica.

Casas, Gerardo; Campos, Ivette; Gutiérrez, Hernán y Smith, Marjorie (2001). La producción del conocimiento de los trabajos finales de graduación y su contribución a las áreas significativas. Universidad de Costa Rica: Sección de Investigación, Escuela de Trabajo Social.

Escuela de Trabajo Social (s.f.). Catálogo de Trabajos Finales Graduación presentados en la Escuela de Trabajo Social. Universidad de Costa Rica, Biblioteca Eugenio Fonseca Tortós.

Guzmán, Laura (s.f.) Módulos de Investigación Avanzada en Trabajo Social Maestria en Trabajo Social. Universidad de Costa Rica, Escuela de Trabajo Social.

Moreno, A. (2000). La Recreación desde la perspectiva de los jóvenes de Liberia. Tesis no publicada, Escuela de Trabajo Social, Universidad de Costa Rica.

Ramírez, L.; Montenegro, L. y Ramírez, C. (2001). El Enfoque alternativo. El manejo de las emociones. Tesis no publicada, Escuela de Trabajo Social, Universidad de Costa Rica.

San Giacomo Parodi, Osvaldo (1997). Trabajo Social. Conocimiento y transformación. Anuario, (2). Departamento de Servicio Social. Universidad Nacional de Mar del Plata. Argentina. 
Sección de Investigación, Escuela de Trabajo Social (2001). Bases de Datos sobre Trabajos Finales Graduación, 1996-2001. Escuela de Trabajo Social, Universidad de Costa Rica.

Sección de Investigación, Escuela de Trabajo Social (1994). Plan de Investigación. Universidad de Costa Rica.

Sección de Investigación, Escuela de Trabajo Social (1999). Plan de Investigación Areas Críticas. Universidad de Costa Rica.

Tobón, Maria Cecilia y otros (1983). La práctica profesional del Trabajo Social. Lima, Perú: CELATS. 